\title{
Model independent analysis of the forward-backward asymmetry of top quark production at the Tevatron
}

\author{
Dong-Won Jung \\ Physics Department and CMTP, National Central University, Jhongli, Taiwan, 32054 \\ P. Ko \\ School of Physics, KIAS, Seoul 130-722, Korea \\ Jae Sik Lee \\ Physics Division, National Center for Theoretical Sciences, Hsinchu, Taiwan 300 \\ Soo-hyeon Nam \\ Korea Institute of Science and Technology Information, Daejeon 305-806, Korea
}

(Dated: December 8, 2009)

\begin{abstract}
Motivated by a possible anomaly in the forward-backward (FB) asymmetry of top quark $\left(A_{\mathrm{FB}}\right)$ observed at the Tevatron, we perform a model independent analysis on $q \bar{q} \rightarrow t \bar{t}$ using an effective lagrangian with dim- 6 four-quark operators. We derive necessary conditions on new physics structures and the couplings that are consistent with the $t \bar{t}$ production cross section and $A_{\mathrm{FB}}$ measured at the Tevatron, and discuss possible new physics scenarios that could generate such dim- 6 operators.
\end{abstract}

1. Top physics has entered a new era after its first discovery, due to the high luminosity achieved at the Tevatron. Most recent results on the top mass and the $t \bar{t}$ production cross section (CDF and D0 Collaborations combined analysis) are: $m_{t}=(171.3 \pm 1.3) \mathrm{GeV}$ and $\sigma_{t \bar{t}}=(7.50 \pm 0.48) \mathrm{pb}$, respectively [1] .

The forward-backward asymmetry $A_{\mathrm{FB}}$ of the top quark is one of the interesting observables related with top quark. Within the Standard Model (SM), this asymmetry vanishes at leading order in QCD because of $C$ symmetry. At next-to-leading order $\left[O\left(\alpha_{s}^{3}\right)\right]$, a nonzero $A_{\mathrm{FB}}$ can develop from the interference between the Born amplitude and two-gluon intermediate state, as well as the gluon bremsstrahlung and gluon-(anti)quark scattering into $t \bar{t}$, with the prediction $A_{\mathrm{FB}} \sim 0.078$ 2]. The measured asymmetry has been off the SM prediction by $2 \sigma$ for the last few years, albeit a large experimental uncertainties. The most recent measurement in the $t \bar{t}$ rest frame is [1]

$A_{\mathrm{FB}} \equiv \frac{N_{t}(\cos \theta \geq 0)-N_{\bar{t}}(\cos \theta \geq 0)}{N_{t}(\cos \theta \geq 0)+N_{\bar{t}}(\cos \theta \geq 0)}=(0.24 \pm 0.13 \pm 0.04)$

with $\theta$ being the polar angle of the top quark with respect to the incoming proton in the $t \bar{t}$ rest frame. This $\sim 2 \sigma$ deviation stimulated some speculations on new physics scenarios 33 .

On the other hand, search for a new resonance decaying into $t \bar{t}$ pair has been carried out at the Tevatron. As of now, there is no clear signal for such a new resonance [1]. Therefore, in this letter, we assume that a new physics scale relevant to $A_{\mathrm{FB}}$ is large enough so that production of a new particle is beyond the reach of the Tevatron, which makes a key difference between our work and other literatures on this subject [3 $[9]$. Then it is ade- quate to integrate out the heavy fields, and we can adopt a model independent effective lagrangian approach in order to study new physics effects on $\sigma_{t \bar{t}}$ and $A_{\mathrm{FB}}$. The resulting effective lagrangian will be an infinite tower of local operators containing light quark $(q=u, d, s, c, b)$, (anti)top quark $(t$ and $\bar{t}$ ) and gluon fields. At the Tevatron, the $t \bar{t}$ production is dominated by $q \bar{q} \rightarrow t \bar{t}$, and it would sufficient to consider dimension- 6 four-quark operators (the so-called contact interaction terms) to describe the new physics effects on the $t \bar{t}$ production at the Tevatron. Note that similar approach was adopted for the dijet production to constrain the composite scale of light quarks, and we are proposing the same analysis for the $t \bar{t}$ system. We will also speculate underlying new physics which could induce the relevant dim- 6 operators in the second half of this work.

2. If new physics scale is high enough, then their effects on the $t \bar{t}$ production at the Tevatron can be described by dim- 6 effective lagrangian. Since the $S U(3)_{C} \times S U(2)_{L} \times U(1)_{Y}$ symmetry has been well established for the light quark system, we assume that $S U(2)_{L} \times U(1)_{Y}$ symmetry is linearly realized on the light quark system. And we impose the custodial symmetry $S U(2)_{R}$ for the light quark sector. Under these assumptions, the dimension- 6 operators relevant to the $t \bar{t}$ production at the Tevatron are

$$
\begin{aligned}
\mathcal{L}_{6}=\frac{g_{s}^{2}}{\Lambda^{2}} \sum_{A, B} & {\left[C_{1 q}^{A B}\left(\bar{q}_{A} \gamma_{\mu} q_{A}\right)\left(\bar{t}_{B} \gamma^{\mu} t_{B}\right)\right.} \\
& \left.+C_{8 q}^{A B}\left(\bar{q}_{A} T^{a} \gamma_{\mu} q_{A}\right)\left(\bar{t}_{B} T^{a} \gamma^{\mu} t_{B}\right)\right]
\end{aligned}
$$

where $T^{a}=\lambda^{a} / 2,\{A, B\}=\{L, R\}$, and $L, R \equiv$ $\left(1 \mp \gamma_{5}\right) / 2$ with $q=(u, d)^{T},(c, s)^{T}$ [18]. Using this effective lagrangian, we calculate the cross section up to 
$O\left(1 / \Lambda^{2}\right)$, keeping only the interference term between the $\mathrm{SM}$ and new physics contributions. In this case, we can safely neglect the flavor changing dim- 6 operators such as $\overline{d_{R}} \gamma^{\mu} s_{R} \overline{t_{R}} \gamma_{\mu} t_{R}$, because they do not interfere with the SM QCD amplitude.

The above effective lagrangian was also discussed in Ref. [10], where the $t$ quark was treated as $S U(2)_{L} \times$ $S U(2)_{R}$ singlet and top currents were decomposed into vector and axial vector currents, rather than chirality basis as in our case. If we include $t_{L}$ in (2), we better work in terms of $Q_{L}^{3} \equiv\left(t_{L}, b_{L}\right)^{T}$, since they forms an $S U(2)_{L}$ doublet. Then we have to consider $b \bar{b}$ too. Note that the $b \bar{b}$ cross section agrees well with the SM predictions, and there are no data on the FB asymmetry of bottom quark at hadron colliders. We can avoid the complication from $b \bar{b}$ system, assuming that only $t_{R}$ appears in the above effective lagrangian. In fact, there are a number of new physics scenarios, where $t_{R}$ (and/or Higgs) is (partially) composite 11]. Then among the dim- 6 four-quark operators, those who have $t_{R}$ and $\bar{t}_{R}$ would be most important at low energy. The case of composite $t_{R}$ can be addressed by choosing $C_{1 q}^{L L}=C_{1 q}^{R L}=C_{8 q}^{L L}=C_{8 q}^{R L}=0$

3. It is straightforward to calculate the amplitude for

$$
q\left(p_{1}\right)+\bar{q}\left(p_{2}\right) \rightarrow t\left(p_{3}\right)+\bar{t}\left(p_{4}\right)
$$

using the above effective lagrangian and the SM. The squared amplitude summed (averaged) over the final (initial) spins and colors is given by

$$
\overline{|\mathcal{M}|^{2}} \simeq \frac{4 g_{s}^{4}}{9 \hat{s}^{2}}\left\{2 m_{t}^{2} \hat{s}\left[1+\frac{\hat{s}}{2 \Lambda^{2}}\left(C_{1}+C_{2}\right)\right] s_{\hat{\theta}}^{2}+\frac{\hat{s}^{2}}{2}\left[\left(1+\frac{\hat{s}}{2 \Lambda^{2}}\left(C_{1}+C_{2}\right)\right)\left(1+c_{\hat{\theta}}^{2}\right)+\hat{\beta}_{t}\left(\frac{\hat{s}}{\Lambda^{2}}\left(C_{1}-C_{2}\right)\right) c_{\hat{\theta}}\right]\right\}
$$

where $\hat{s}=\left(p_{1}+p_{2}\right)^{2}, \hat{\beta}_{t}^{2}=1-4 m_{t}^{2} / \hat{s}$, and $s_{\hat{\theta}} \equiv \sin \hat{\theta}$ and $c_{\hat{\theta}} \equiv \cos \hat{\theta}$ with $\hat{\theta}$ being the polar angle between the incoming quark and the outgoing top quark in the $t \bar{t}$ rest frame. And the couplings are defined as: $C_{1} \equiv$ $C_{8 q}^{L L}+C_{8 q}^{R R}$ and $C_{2} \equiv C_{8 q}^{L R}+C_{8 q}^{R L}[19$. Since we have kept only up to the interference terms, there are no contributions from the color-singlet operators with coupling $C_{1 q}^{A B}$. The first (second) term is for the production of top quarks with the same (different) helicities. The term linear in $\cos \hat{\theta}$ could generate the forward-backward asymmetry which is proportional to $\Delta C \equiv\left(C_{1}-C_{2}\right)$. Note that both light quark and top quark should have chiral couplings to the new physics in order to generate $A_{\mathrm{FB}}$ at the tree level (namely $\Delta C \neq 0$ ). Therefore there could be parity violation in the four-quark operators with light quarks only, depending on the underlying physics. Since the SM neutral weak current effect is order of $g_{2}^{2} / m_{Z}^{2}$, it would be safe if $g_{s}^{2} C / \Lambda^{2} \lesssim g_{2}^{2} / m_{Z}^{2}$, which is not so difficult to achieve. This parity violation, if large, could be observed in the nonzero (anti)top spin polarization [12].

We convolute the parton level cross section with CTEQ6L parton distribution functions with factorization and renormalization scale equal to $m_{t}=172.5 \mathrm{GeV}$ in order to get the cross sections and compared with the data from the Tevatron. The QCD corrections are taken into account by the $K$ factor $(K=1.3)$ for the SM contributions in the total cross section. When we calculate the FB asymmetry, we use the LO results for the cross section.

In order to estimate the validity of our description based on effective lagrangian up to dimension 6 operators, we have to estimate the effects of the ignored dim- 8 operators to the $t \bar{t}$ production at the Tevatron. For this purpose, we calculate the amplitude squared from the effective lagrangian (2), see Appendix, and consider it as the rough estimate of dim- 8 operators, by comparing it to its interference term with the SM amplitude as well as to the SM amplitude squared. We note that, without considering all the possible dim- 8 operators explicitly, the criterion adopted here for the validity of our description should not be unambiguous, and intrinsically qualitative rather than quantitative.

We find that the amplitude squared of dim- 6 operators does not show simple dependence on the Wilson coefficients as the interference term. Moreover, it includes additional contribution from the color-singlet operator which is proportional to $\left(C_{1 q}^{A B}\right)^{2}$ and enhanced by the factor $9 / 2$ compared to the color-octet contribution. Since the color-singlet operators do not interfere with the SM ones, we set $C_{1 q}^{A B}=0$ and consider the validity region of our approach.

There are 3 inequalities we can consider:

$$
\begin{aligned}
\sigma_{\text {int }} & <r \sigma_{\mathrm{SM}}, \\
\sigma_{\mathrm{NP}} & <r \sigma_{\mathrm{int}}, \\
\sigma_{\mathrm{NP}} & <r^{2} \sigma_{\mathrm{SM}},
\end{aligned}
$$

where $\sigma_{\text {int }}$ is the interference between the SM and the dim- 6 operators, and $\sigma_{\mathrm{NP}}$ is the contributions solely from the dim- 6 operators. The parameter $r$, a kind of fuzzy factor, measures the ratio of the subleading term to the leading $\sigma_{\mathrm{SM}}$ (SM contribution), and we take $r=0.3,0.5$ and 1.0 in the following. We vary the couplings $C_{8 q}^{A B}$ in the range between -10 and +10 , and find the regions that satisfy the above inequalities. 


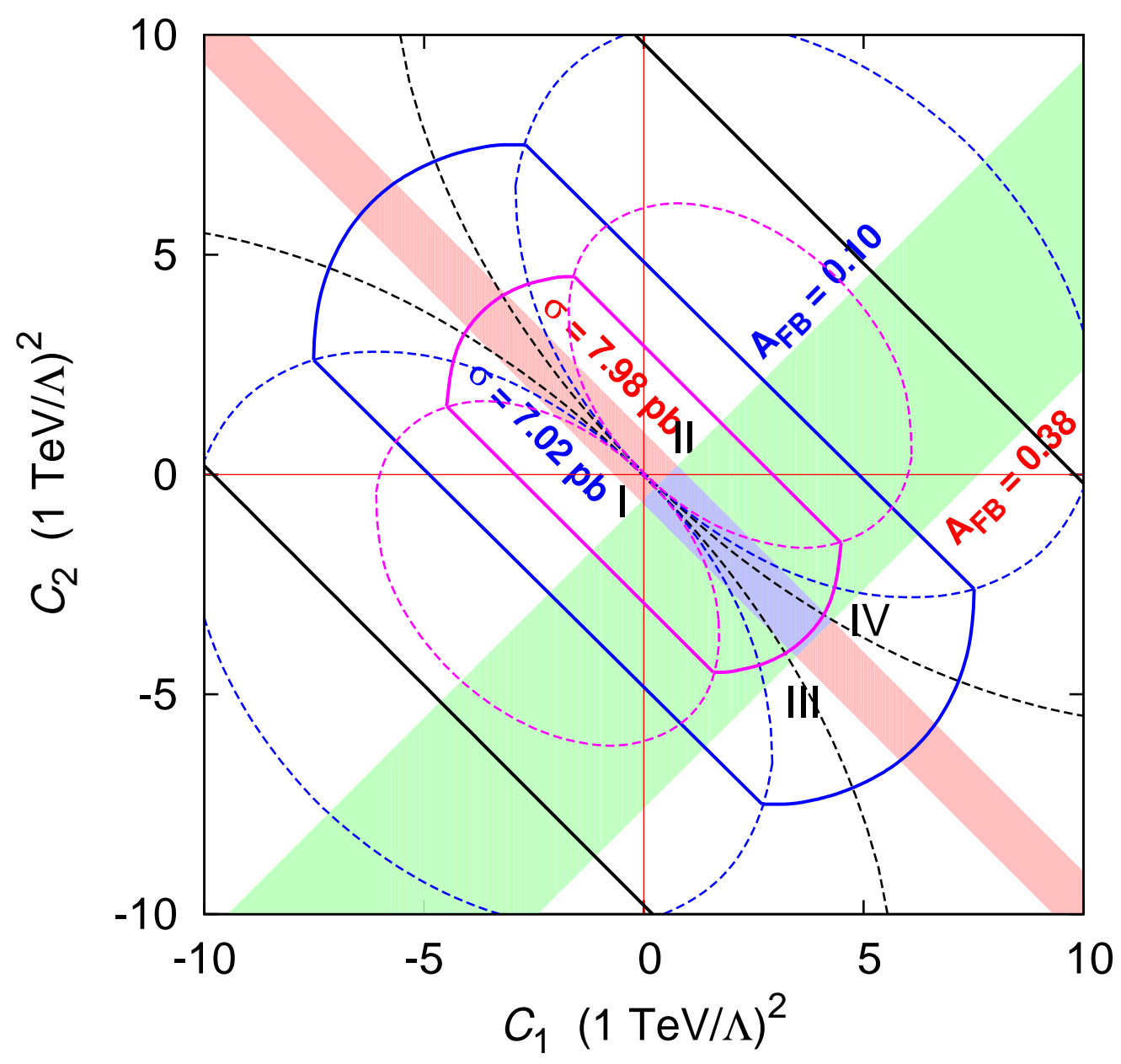

FIG. 1: The region in $\left(C_{1}, C_{2}\right)$ plane that is consistent with the Tevatron data at the $1 \sigma$ level: $\sigma_{t \bar{t}}=(7.50 \pm 0.48)$ pb and $A_{\mathrm{FB}}=(0.24 \pm 0.13 \pm 0.04)$. Also shown are the boundaries of the validity regions described in the text taking $r=0.3$ (magenta), 0.5 (blue) and 1 (black). The straight lines are from the inequality Eq. (4), the circles and their mirror images from the inequality Eq. (5), and the ellipses centered at the origin from Eq. (6).

The first inequality constrains the parameter space to the band around $C_{1}+C_{2}=0$. The 2 nd relation implies that the contributions from dim- 8 operators are smaller than the dim- 6 operators, and should be a good estimate in most cases. However, in our case, the interference $\sigma_{\text {int }}$ is vanishing along the line $C_{1}+C_{2}=0$, and we can not rely on the inequality, Eq. (5). Therefore we also impose the 3rd inequality, Eq. (6), which is relevant when $\sigma_{\mathrm{NP}} \propto\left(C_{1}+C_{2}\right) \approx 0$, and the constraint is the elliptical shape centered at the origin. Note that we impose the inequalities either (5) or (6) as the validity region, since we cannot apply the inequality (5) around the line $C_{1}+$ $C_{2}=0$.

In Fig. 11 we show the allowed region in the $\left(C_{1}, C_{2}\right)$ plane that is consistent with the Tevatron data at the $1 \sigma$ level: $\sigma_{t \bar{t}}=(7.50 \pm 0.48) \mathrm{pb}$ and $A_{\mathrm{FB}}=(0.24 \pm 0.13 \pm$ 0.04). The allowed region is around $0 \lesssim C_{1} \lesssim 4$ and
$-4 \lesssim C_{2} \lesssim+0.5$. The negative sign of $C_{2}$ is preferred at the $1 \sigma$ level. The validity region described in the previous paragraph is indicated in magenta, blue, black for $r=0.3,0.5$ and 1 , respectively. The straight line is from the inequality Eq. (4), the circles and their mirror images are from the inequality Eq. (5), and the ellipse centered at the origin is from Eq. (6). Note that our effective lagrangian approach based on dim- 6 operators is valid in an ample parameter space.

In Fig. 2 (a), we show the $\sqrt{\hat{s}}=M_{t \bar{t}}$ distributions for four different values of $\left(C_{1}, C_{2}\right)$ corresponding to the four corners of the allowed region in Fig. 1 as well as the SM prediction. The $M_{t \bar{t}}$ distribution depends only on $\sigma_{t \bar{t}}$, and there is no informations on the chiral structure because we have integrated out the angular dependence as well as new heavy degrees of freedom that could modify $q \bar{q} \rightarrow t \bar{t}$. 

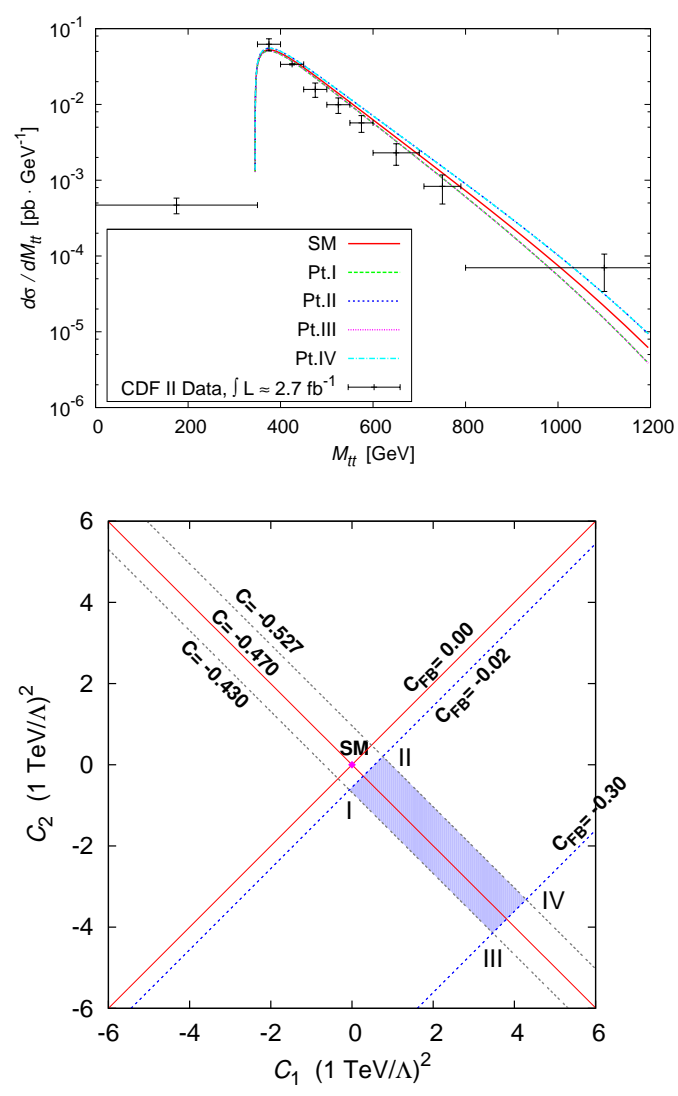

FIG. 2: (a) The $M_{t \bar{t}}$ distribution for the points I,II,III, IV and the SM and (b) the spin-spin correlations $C$ and $C_{F B}$.

4. Another interesting observable which is sensitive to the chiral structure of new physics affecting $q \bar{q} \rightarrow t \bar{t}$ is the top quark spin-spin correlation [13]:

$$
C=\frac{\sigma\left(t_{L} \bar{t}_{L}+t_{R} \bar{t}_{R}\right)-\sigma\left(t_{L} \bar{t}_{R}+t_{R} \bar{t}_{L}\right)}{\sigma\left(t_{L} \bar{t}_{L}+t_{R} \bar{t}_{R}\right)+\sigma\left(t_{L} \bar{t}_{R}+t_{R} \bar{t}_{L}\right)} .
$$

This quantity depends on the spin quantization axis, and there are three different axes that are mostly used: the beam axis, the top direction (helicity basis), and the offdiagonal axis. At leading order, the SM prediction is $C=-0.471$ for the helicity basis which we choose in this work. It is known that NLO correction to $C$ is rather large, shifting $C$ to -0.352 [13], but it is beyond the scope of this work to pursue this issue further here.

Recently the top spin-spin correlation was measured by both CDF and D0 Collaborations at the Tevatron [14]. Using the off-diagonal axis as the quantization axis in order to maximize the spin-spin correlation, they found $C=0.32_{-0.78}^{+0.55}$ (CDF dilepton mode), $C=0.60 \pm$ 0.50 (stat) \pm 0.16 (syst) (CDF lepton + jets mode), and $C=-0.17_{-0.53}^{+0.64}$ (D0 dilepton mode). The data are consistent with the SM prediction $C=0.78$ (for the off-diagonal basis), albeit rather large uncertainties. It would be interesting if the SM prediction is confirmed or not with more accumulated data in the future.
Since new physics must have chiral couplings both to light quarks and top quark, the spin-spin correlation defined above will be affected. From Eqs. (3) and (7), it is clear this correlation is sensitive to $\left(C_{1}+C_{2}\right)$, since the linear term in $\cos \hat{\theta}$ does not contribute to the correlation $C$ after integration over $\cos \hat{\theta}$. On the other hand, if one considers the forward and the backward regions separately, the spin-spin correlation would depend on $\left(C_{1}-C_{2}\right)$ and will be closely correlated with $A_{\mathrm{FB}}$. Therefore we propose a new spin-spin FB asymmetry $C_{F B}$ defined as

$$
C_{F B} \equiv C(\cos \theta \geq 0)-C(\cos \theta \leq 0),
$$

where $C(\cos \theta \geq 0(\leq 0))$ implies the cross sections in the numerator of Eq. (7) are obtained for the forward (backward) region: $\cos \theta \geq 0(\leq 0)$. This quantity can be measured by dividing the $t \bar{t}$ sample into the forward top and the backward top events. In Fig. 2 (b), we show the contour plots for the $C$ and $C_{F B}$ in the $\left(C_{1}, C_{2}\right)$ plane along with the SM prediction at LO. Note that there is a clear correlation between $C_{F B}$ and $A_{F B}$ in Fig. 1 which must be observed in the future measurements if the $A_{\mathrm{FB}}$ anomaly is real and a new particle is too heavy to be produced at the Tevatron.

5. So far, we considered dim- 6 four-quark operators that could affect the $t \bar{t}$ productions at the Tevatron, and found the necessary conditions for accommodating $A_{\text {FB }}$. Now we study specific new physics that could generate the relevant dim- 6 operators with corresponding Wilson coefficients. It is impossible to exhaust all the possibilities, and we consider the following interactions of quarks with spin-1 flavor-conserving (FC) color-octet $V_{8}^{a}$ vectors, spin-1 flavor-violating $(\mathrm{FV})$ color-singlet $\tilde{V}_{1}$ and color-octet $\tilde{V}_{8}^{a}$ vectors, and spin-0 FV color-singlet $\tilde{S}_{1}$ and color-octet $\tilde{S}_{8}^{a}$ scalars $(A=L, R)$ :

$$
\begin{aligned}
& \mathcal{L}_{\text {int }}=g_{s} V_{8}^{a \mu} \sum_{A}\left[g_{8 q}^{A}\left(\bar{q}_{A} \gamma_{\mu} T^{a} q_{A}\right)+g_{8 t}^{A}\left(\bar{t}_{A} \gamma_{\mu} T^{a} t_{A}\right)\right] \\
& +g_{s}\left[\tilde{V}_{1}^{\mu} \sum_{A} \tilde{g}_{1 q}^{A}\left(\bar{t}_{A} \gamma_{\mu} q_{A}\right)+\tilde{V}_{8}^{a \mu} \sum_{A} \tilde{g}_{8 q}^{A}\left(\bar{t}_{A} \gamma_{\mu} T^{a} q_{A}\right)+\text { h.c. }\right] \\
& +g_{s}\left[\tilde{S}_{1} \sum_{A} \tilde{\eta}_{1 q}^{A}(\bar{t} A q)+\tilde{S}_{8}^{a} \sum_{A} \tilde{\eta}_{8 q}^{A}\left(\bar{t} A T^{a} q\right)+\text { h.c. }\right],
\end{aligned}
$$

where $q$ denotes light quarks (either $u$ or $d$ depending on the models). This interaction lagrangian encompasses many models beyond the SM, and makes a good starting point to study the underlying mechanism for the effective lagrangian discussed earlier. If the spin-1 particle has both the FC and FV interactions, we set $V_{8}^{\mu}=\tilde{V}_{8}^{\mu}$. The axigluon model [15] corresponds to flavor universal chiral couplings: $g_{8 q}^{L}=g_{8 t}^{L}=-g_{8 q}^{R}=-g_{8 t}^{R}=1$. The model in Ref. [6] has a new gauge boson $Z^{\prime}$ with dominant couplings to the $u$ and $t$ quarks, which in our language corresponds to $\tilde{V}_{1}=Z^{\prime}$ and $g_{s} \tilde{g}_{1 q}^{R}=g_{X}$ ignoring the FC coupling. The model in Ref. 7] has a new charged gauge 
boson $W^{ \pm^{\prime}}$, which is obtained in our case by $\tilde{V}_{1}=W^{\prime}$ and $g_{s} \tilde{g}_{1 q}^{A}=g^{\prime} g_{A}$ for $A=L$ or $R$, respectively. In some $\mathrm{RS}$ scenarios, one can have large flavor mixing in the right-handed quark sector [16]: $g_{8 q}^{L}=g_{8 q}^{R}=g_{8 b}^{R} \simeq-0.2$, $g_{8 t}^{L}=g_{8 b}^{L} \simeq(1 \sim 2.8), g_{8 t}^{R} \simeq(1.5 \sim 5), \tilde{g}_{8 q}^{L} \simeq V_{t q}$ and $\tilde{g}_{8 q}^{R} \simeq 1$.

After integrating out the heavy vector and scalar fields, we obtain the Wilson coefficients as follows:

$$
\begin{aligned}
& \frac{C_{8 q}^{L L}}{\Lambda^{2}}=-\frac{1}{m_{V}^{2}} g_{8 q}^{L} g_{8 t}^{L}-\frac{1}{m_{\tilde{V}}^{2}}\left[2\left|\tilde{g}_{1 q}^{L}\right|^{2}-\frac{1}{N_{c}}\left|\tilde{g}_{8 q}^{L}\right|^{2}\right], \\
& \left.\frac{C_{8 q}^{R R}}{\Lambda^{2}}=-\frac{1}{m_{V}^{2}} g_{8 q}^{R} g_{8 t}^{R}-\frac{1}{m_{\tilde{V}}^{2}}\left[2\left|\tilde{g}_{1 q}^{R}\right|^{2}-\frac{1}{N_{c}}\left|\tilde{g}_{8 q}^{R}\right|^{2}\right], 10\right) \\
& \frac{C_{8 q}^{L R}}{\Lambda^{2}}=-\frac{1}{m_{V}^{2}} g_{8 q}^{L} g_{8 t}^{R}-\frac{1}{m_{\tilde{S}}^{2}}\left[\left|\tilde{\eta}_{1 q}^{L}\right|^{2}-\frac{1}{2 N_{c}}\left|\tilde{\eta}_{8 q}^{L}\right|^{2}\right], \\
& \frac{C_{8 q}^{R L}}{\Lambda^{2}}=-\frac{1}{m_{V}^{2}} g_{8 q}^{R} g_{8 t}^{L}-\frac{1}{m_{\tilde{S}}^{2}}\left[\left|\tilde{\eta}_{1 q}^{R}\right|^{2}-\frac{1}{2 N_{c}}\left|\tilde{\eta}_{8 q}^{R}\right|^{2}\right],
\end{aligned}
$$

where $m_{V}\left(m_{\tilde{V}}\right)$ and $m_{\tilde{S}}$ denote the masses of vectors $V_{8}\left(\tilde{V}_{i}\right)$ and scalars $\tilde{S}_{i}(i=1,8)$, respectively.

Another interesting possibility is minimal flavor violating interactions of color-triplet $S_{k}^{\gamma}$ with mass $m_{S_{3}}$ and color-sextet scalars $S_{i j}^{\alpha \beta}$ with mass $m_{S_{6}}$ with the SM quarks [17]. Here $\alpha, \beta, \gamma$ and $i, j, k$ are color and flavor indices, respectively. For example, if we consider the following interactions (Model V and VI in Ref. [17]),

$$
\mathcal{L}=g_{s}\left[\frac{\eta_{3}}{2} \epsilon_{\alpha \beta \gamma} \epsilon^{i j k} u_{i R}^{\alpha} u_{j R}^{\beta} S_{k}^{\gamma}+\eta_{6} u_{i R}^{\alpha} u_{j R}^{\beta} S_{i j}^{\alpha \beta}+\text { h.c. }\right]
$$

the $u$-channel exchange of new scalars can contribute to $u \bar{u} \rightarrow t \bar{t}$, resulting in [20]

$$
\frac{C_{8 u}^{R R}}{\Lambda^{2}}=-\frac{\left|\eta_{3}\right|^{2}}{m_{S_{3}}^{2}}+\frac{2\left|\eta_{6}\right|^{2}}{m_{S_{6}}^{2}} .
$$

Since these new scalars couple only to the right-handed up-type quarks, constraints on the couplings $\eta_{3}$ and $\eta_{8}$ from flavor physics are rather weak, and one can accommodate the observed $A_{\mathrm{FB}}$ easily.

In Table @ we show the new particle exchanges under consideration and the couplings $C_{1}, C_{2}$ induced by them. We found that the four types of exchanges of $V_{8}, \tilde{V}_{8}$, $\tilde{S}_{1}$, and $S_{13}^{\alpha \beta}$ could give rise to the large positive $A_{\mathrm{FB}}$ at the 1- $\sigma$ level. Among them, the following three types of couplings are more or less fixed as:

$$
\begin{aligned}
\tilde{V}_{8} & : \frac{1}{N_{c}}\left(\frac{1 \mathrm{TeV}}{m_{\tilde{V}}}\right)^{2}\left(\left|\tilde{g}_{8 q}^{L}\right|^{2}+\left|\tilde{g}_{8 q}^{R}\right|^{2}\right) \simeq 0.76, \\
\tilde{S}_{1} & :\left(\frac{1 \mathrm{TeV}}{m_{\tilde{S}}}\right)^{2}\left(\left|\tilde{\eta}_{1 q}^{L}\right|^{2}+\left|\tilde{\eta}_{1 q}^{R}\right|^{2}\right) \simeq 0.62, \\
S_{13}^{\alpha \beta} & : 2\left(\frac{1 \mathrm{TeV}}{m_{S_{6}}}\right)^{2}\left|\eta_{6}\right|^{2} \simeq 0.76 .
\end{aligned}
$$

For the case of $V_{8}$, Fig. 3] shows the 1- $\sigma$ favored regions of the couplings in the $\left(g_{8 t}^{L}, g_{8 t}^{R}\right)$ plane for several choices of $r_{q} \equiv g_{8 q}^{R} / g_{8 q}^{L}$ taking $g_{8 q}^{L}=1$. We observe that the regions lie along the direction of $g_{8 t}^{L}=-g_{8 t}^{R}$ and $g_{8 t}^{L} / g_{8 q}^{L}$ tends to be positive (negative) for $r_{q}>1\left(r_{q}<1\right)$. Note that $r_{q}=1$ is not allowed because $\Delta C=0$ and the constraint from $\sigma_{t \bar{t}}$ disappears when $r_{q}=-1$, since $C_{1}+C_{2}=0$. It would be interesting to search for new vector or scalar particles that satisfy the above conditions at LHC.

A caution is in order. The second row with $\tilde{V}_{1}$ in Table I is shown to be disfavored, which seems to be contradictory to the results of Refs. [6, 7] where new particles were assumed to be light. However this is not the case, since we took a heavy new particle limit ( far below the new particle mass scale, $\left.\hat{s}, \hat{t} \ll m_{\tilde{V}}^{2}\right)$ in order to derive $C_{8 q}^{A B}$ 's, though we began with the full amplitudes using the above interaction lagrangian which are the same as the amplitudes in Refs. [6, 7]. Therefore our results can not be in conflict with the claims of Refs. [6, 7]. Rather, our results simply imply that a color-singlet spin-1 object cannot accommodate the $A_{\mathrm{FB}}$ if it is too heavy to be directly produced at the Tevatron. In other words, one has to consider a light $\tilde{V}_{1}$ scenario, where $\tilde{V}_{1}$ has to be light enough $(\sim O(100) \mathrm{GeV})$ as in Refs. [6, 7], and then our effective lagrangian approach is not applicable for such a light $\tilde{V}_{1}$.

On the other hand, a new particle with different quantum numbers (color, spin and flavor mixings) can affect the $A_{\mathrm{FB}}$, even if it is too heavy to be produced directly at the Tevatron. As an example, one can consider the color-sextet case in order to make clear our point. In this case, only $C_{8 u}^{R R}$ (and $C_{1 u}^{R R}$ which is irrelevant in the interference) is nonzero, and we have $C_{2}=0$. Then Fig. 1 indicates that a very heavy color-sextet can enhance $A_{\mathrm{FB}}$ roughly up to $\sim 12 \%$ at the $1 \sigma$ deviation in the total cross section $\sigma_{t \bar{t}}$ to the larger value. For the color antitriplet case, the signs of $C_{8 u}^{R R}$ and $C_{1}$ are opposite to the color sextet case [ see Eq. (12) ], and the resulting $A_{\mathrm{FB}}$ is less than the color-sextet case, with affecting $\sigma_{t \bar{t}}$ in the lower value. These tendency can be shown to be consistent with the calculations based on the full amplitudes [6, 7, 12]. In order to increase $A_{\mathrm{FB}}$ more, there would be a strong tension with the total cross section $\sigma_{t \bar{t}}$, if a new physics scale is beyond the reach of Tevatron. However this tension might be avoided (or lessened) if the new particle is light enough, and we should not rely on the effective lagrangian approach for this case. One can understand other entries of Table I in the similar fashion.

For more quantitative discussions, we have to study the full amplitude and compare the results with our effective lagrangian approach, and investigate the validity region of the effective lagrangian approach model by model. This is clearly beyond the scope of the present work, and the detailed study will be presented in the future work 12 .

6. In this letter, we performed a model independent study of $t \bar{t}$ productions at the Tevatron using dimension- 
TABLE I: New particle exchanges and the signs of induced couplings $C_{1}$ and $C_{2}$

\begin{tabular}{l|c|c|c|c}
\hline \hline New particles & couplings & $C_{1}$ & $C_{2}$ & $1 \sigma$ favor \\
\hline \hline$V_{8}$ (spin-1 FC octet) & $g_{8 q, 8 t}^{L, R}$ & indef. & indef. & $\sqrt{ }$ \\
$\tilde{V}_{1}$ (spin-1 FV singlet) & $\tilde{g}_{1 q}^{L, R}$ & - & 0 & $\times$ \\
$\tilde{V}_{8}$ (spin-1 FV octet) & $\tilde{g}_{8 q}^{L, R}$ & + & 0 & $\sqrt{ }$ \\
\hline$\tilde{S}_{1} \quad$ (spin-0 FV singlet) & $\tilde{\eta}_{1 q}^{L, R}$ & 0 & - & $\sqrt{ }$ \\
$\tilde{S}_{8} \quad$ (spin-0 FV octet) & $\tilde{\eta}_{8 q}^{L, R}$ & 0 & + & $\times$ \\
$S_{2}^{\alpha} \quad$ (spin-0 FV triplet) & $\eta_{3}$ & - & 0 & $\times$ \\
$S_{13}^{\alpha \beta} \quad$ (spin-0 FV sextet) & $\eta_{6}$ & + & 0 & $\sqrt{ }$ \\
\hline \hline
\end{tabular}

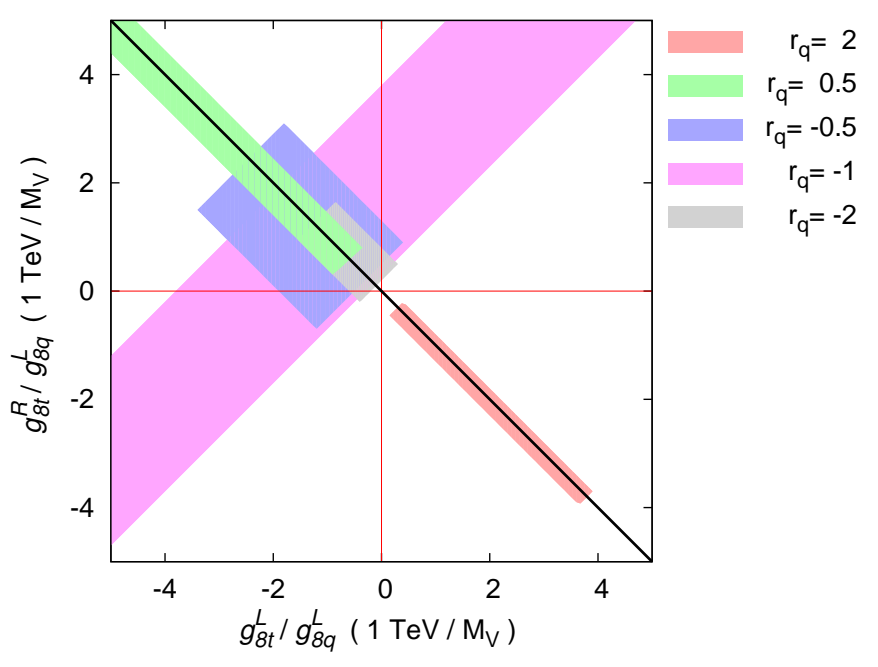

FIG. 3: The 1- $\sigma$ favored region of the couplings for the exchanges of the spin-1 FC color-octet vector bosons $V_{8}$.
6 contact interactions relevant to $q \bar{q} \rightarrow t \bar{t}$. We derived conditions for the couplings of four-quark operators that could generate the FB asymmetry observed at the Tevatron [Fig. 1]. Then we considered the $s-, t-$ and $u$-channel exchanges of spin- 0 and spin- 1 particles whose color quantum number is either singlet, octet, triplet or sextet. Our results in Eqs. (10), (12), and (13) and Fig. 3 encode the necessary conditions for the underlying new physics in a compact and an effective way, when those new particles are too heavy to be produced at the Tevatron but still affect $A_{\mathrm{FB}}$. If these new particles could be produced directly at the Tevatron or at the LHC, we cannot use the effective lagrangian any more. We have to study specific models case by case, and anticipate rich phenomenology at colliders as well as at low energy. Detailed study of these issues lies beyond the scope of this letter, and will be discussed in the future publications [12].

We are grateful to S. Choi, D.H. Kim, S.B. Kim and I. Yu for useful communications, and S.C.Park for collaboration in the initial stage of this work. Part of works by PK were done at Aspen Center for Physics, and Galileo Galilei Institute for Theoretical Physics. The work of Nam was supported in part by Basic Science Research Program through the National Research Foundation of Korea (NRF) funded by the Ministry of Education, Science and Technology (2009-0086961).

\section{Appendix}

In this Appendix, we present the explicit form of the amplitude squared from the effective lagrangian (2), which is used to calculate $\sigma_{\mathrm{NP}}$, as follows:

$$
\begin{aligned}
\overline{\left|\mathcal{M}_{\mathrm{NP}}\right|^{2}}=\frac{4 g_{s}^{4}}{9 \hat{s}^{2}} \frac{\hat{s}^{2}}{4 \Lambda^{4}} & \left\{\left[9\left(\left(C_{1 q}^{L L}\right)^{2}+\left(C_{1 q}^{R R}\right)^{2}\right)+2\left(\left(C_{8 q}^{L L}\right)^{2}+\left(C_{8 q}^{R R}\right)^{2}\right)\right]\left(\hat{u}-m_{t}^{2}\right)^{2}\right. \\
+ & {\left[9\left(\left(C_{1 q}^{R L}\right)^{2}+\left(C_{1 q}^{L R}\right)^{2}\right)+2\left(\left(C_{8 q}^{R L}\right)^{2}+\left(C_{8 q}^{L R}\right)^{2}\right)\right]\left(\hat{t}-m_{t}^{2}\right)^{2} } \\
+ & {\left.\left[9\left(C_{1 q}^{L L} C_{1 q}^{L R}+C_{1 q}^{R R} C_{1 q}^{R L}\right)+2\left(C_{8 q}^{L L} C_{8 q}^{L R}+C_{8 q}^{R R} C_{8 q}^{R L}\right)\right]\left(2 \hat{s} m_{t}^{2}\right)\right\}, }
\end{aligned}
$$

where $\hat{u}-m_{t}^{2}=-\hat{s}\left(1+\hat{\beta}_{t} c_{\hat{\theta}}\right) / 2$ and $\hat{t}-m_{t}^{2}=-\hat{s}\left(1-\hat{\beta}_{t} c_{\hat{\theta}}\right) / 2$.

[1] http://www-cdf.fnal.gov/physics/new/top/top.html

[2] J. H. Kuhn and G. Rodrigo, Phys. Rev. Lett. 81, 49 (1998); J. H. Kuhn and G. Rodrigo, Phys. Rev. D 59,
054017 (1999); O. Antunano, J. H. Kuhn and G. Rodrigo, Phys. Rev. D 77, 014003 (2008).

[3] D. Choudhury, R. M. Godbole, R. K. Singh and K. Wagh, Phys. Lett. B 657, 69 (2007).

[4] A. Djouadi, G. Moreau, F. Richard and R. K. Singh, arXiv:0906.0604 [hep-ph]. 
[5] P. Ferrario and G. Rodrigo, Phys. Rev. D 80, 051701 (2009).

[6] S. Jung, H. Murayama, A. Pierce and J. D. Wells, arXiv:0907.4112 [hep-ph].

[7] K. Cheung, W. Y. Keung and T. C. Yuan, arXiv:0908.2589 [hep-ph].

[8] P. H. Frampton, J. Shu and K. Wang, arXiv:0911.2955 [hep-ph].

[9] J. Shu, T. M. P. Tait and K. Wang, arXiv:0911.3237 [hep-ph]; A. Arhrib, R. Benbrik and C. H. Chen, arXiv:0911.4875 [hep-ph].

[10] C. T. Hill and S. J. Parke, Phys. Rev. D 49, 4454 (1994).

[11] H. Georgi, L. Kaplan, D. Morin and A. Schenk, Phys. Rev. D 51, 3888 (1995); K. Agashe, R. Contino and R. Sundrum, Phys. Rev. Lett. 95, 171804 (2005); B. Lillie, J. Shu and T. M. P. Tait, JHEP 0804, 087 (2008) arXiv:0712.3057 [hep-ph]]; A. Pomarol and J. Serra, Phys. Rev. D 78, 074026 (2008); K. Kumar, T. M. P. Tait and R. Vega-Morales, JHEP 0905, 022 (2009) arXiv:0901.3808 [hep-ph]].

[12] D.W. Jung, P. Ko, J.S. Lee and S.H. Nam, work in preparation.

[13] G. Mahlon and S. J. Parke, Phys. Rev. D 53, 4886 (1996); T. Stelzer and S. Willenbrock, Phys. Lett. B 374, 169 (1996); W. Bernreuther, A. Brandenburg, Z. G. Si and P. Uwer, Nucl. Phys. B 690, 81 (2004).

[14] U. Heinz, talk at Aspen Particle Physics Conference, Jan.
17-23, 2010, Aspen, USA.

[15] J. C. Pati and A. Salam, Phys. Lett. B 58, 333 (1975); L. J. Hall and A. E. Nelson, Phys. Lett. B 153, 430 (1985); P. H. Frampton and S. L. Glashow, Phys. Lett. B 190, 157 (1987); P. H. Frampton and S. L. Glashow, Phys. Rev. Lett. 58, 2168 (1987); J. Bagger, C. Schmidt and S. King, Phys. Rev. D 37, 1188 (1988).

[16] P. M. Aquino, G. Burdman and O. J. P. Eboli, Phys. Rev. Lett. 98, 131601 (2007); K. Agashe, A. Belyaev, T. Krupovnickas, G. Perez and J. Virzi, Phys. Rev. D 77, 015003 (2008).

[17] J. M. Arnold, M. Pospelov, M. Trott and M. B. Wise, arXiv:0911.2225 [hep-ph].

[18] Although we assume the $S U(2)_{L} \times S U(2)_{R}$ chiral symmetry for light quarks, all the explicit models do not satisfy this condition. In that case, one can interpret $q=u, d, s, c, b$.

[19] Throughout this work, unless explicitly written, we are taking $C_{8 q}^{A B}=C_{8 u}^{A B}=C_{8 d}^{A B}$ assuming the $S U(2)_{L} \times$ $S U(2)_{R}$ chiral symmetry. Under this assumption, the down-quark contribution to $\sigma_{t \bar{t}}$ and $A_{\mathrm{FB}}$ is suppressed relative to the up-quark one by a factor more than $\sim 6$ at the Tevatron.

[20] $C_{1 q}^{R R}$ is also induced by color-triplet and sextet scalars, but is not shown, since it is irrelevant here. 